\title{
The Influence of Allocating the Residual Value of MSMEs' Cluster on the Growth of MSMEs and the Cluster Based on the Theory of Structural Hole
}

\author{
Suo $\mathrm{Lu}^{1}$ \\ ${ }^{1}$ Lecturer, Doctoral Program of Stamford International University, Thailand \\ Correspondence: Suo Lu, Doctoral Program of Stamford International University, Rama 9, Bangkok, Thailand.
}

Received: September 14, 2020

Accepted: October 10, 2020

Online Published: October 21, 2020

doi:10.5539/ibr.v13n11p54

URL: https://doi.org/10.5539/ibr.v13n11p54

\begin{abstract}
The objectives of this research were to explicate the influence mechanism between MSMEs and MSMEs' clusters; to explicate the generation mechanism of cluster residual value, and to determine whether there is a significant effect of structural hole on allocating the residual value of MSMEs' cluster.

The research was designed as quantitative research and used survey questionnaires to collect data from 475 entrepreneurs or senior managers of MSMEs. After passing the validity (KMO) and reliability (Cronbach's Alpha) tests, the correlations between independent and dependent variables have been examined by Pearson Correlation. Then One-Way ANOVA was employed for further specifying the causal direction of correlation between variables. The findings of this research showed that there are positive correlations between cluster's value and growth of MSMEs; between structural hole and allocation of cluster's residual value; between structural hole and growth of cluster. In addition, structural hole as a moderating variable effect on the relationship between cluster's value and growth of MSMEs significantly.
\end{abstract}

Keywords: cluster's residual value, enterprise cluster, MSMEs, Relationship network, structural hole

\section{Introduction}

Enterprise cluster is a group of independent and interdependent member enterprises that maintain long-term non-specific contracts according to the division of labor, resource complementation, and on the basis of human factors such as trust and commitment. (Porter, 1998). Based on this understanding, MSMEs' cluster could be considered as in a specific field, a collection of a large number of interconnected Micro, Small and Medium-sized Enterprises and related supporting institutions with a leading industry as their core business.

With the globalization of market competition, economic development also highlights the trend of regional centralization, particularly on the local enterprise clusters who are participating in the division of labor in the global industrial chain. The prosperity of Silicon Valley, the revival of the Italian economy, and the rapid development of the eastern coastal towns of China are largely attributed by the development of local enterprise clusters. The clusters of MSMEs have been developing rapidly due to its ability to adapt to market changes and meet the personalized needs of customers. The cluster development of MSMEs is an important economic phenomenon in the world, the MSMEs' clusters in China also achieved a booming development in the 1990s, an obvious huge economic and social benefits brought by MESMs' clusters are increasing. Therefore, deeply analyzing the economic phenomenon of MSMEs' Cluster has great significance to promote regional economic development and social progress.

As a major subject group of micro-economic, MSMEs is playing a more and more important role in economic and social development, nowadays. In both developed and developing countries or different economics areas, there are a large number of MSMEs, which play a significant role in promoting local economic and social development. Thus, the development of MSMEs is valued and supported by the government. According to the world bank (2019), Formal MSMEs contribute up to $60 \%$ of total employment and up to $40 \%$ of national income (GDP) in emerging economies, and these numbers are significantly higher when informal MSMEs are included. In 199 economies areas of the world, there are about 322.4 million formal MSMEs, which employs more than 1 billion employees. (MSME-CI, 2019). 
Until 2019, more than 23,280,000 MSMEs have been registered in China, accounting for $99 \%$ of the total number of Industrial and Commercial registration. MSMEs contributed 60 percent of China's GDP, 50 percent of tax revenue, and 80 percent of urban employment. In addition, MSMEs supported more than 65 percent of China's invention patents, 75 percent of enterprise technological innovation, and 80 percent of new product development (Chinabgao, 2019). MSMEs have become the main force to promote the sustained and rapid development of China's economy.

To take a panoramic view of the successful MSMEs in various countries, most of them with fast development, good benefits, and strong vitality do not exist sporadically, but come together in a cluster and benefit a lot from the powerful cluster's effect. Such as the textile industry in northern Italy, the wine industry in California, the IT industry in Silicon Valley, and the automobile industry in Japan. The local economic development in China is also inseparable from the contribution of MSMEs' clusters, such as the leather and shoe industry in Wenzhou, the computer information industry in Dongguan, and textile industry in Foshan.

\subsection{Problem Statement}

The cluster of MSMEs is playing a key role in the new economic structure of China. However, it is still difficult for most MSMEs to draw benefit from the cluster they are in. Although many scholars have discussed the enterprise cluster from different perspectives in previous studies, there is still a large gap in the study of cluster residual value distribution from the perspective of structural hole managing and optimizing. In the previous researches about enterprise cluster, both of the knowledge school represented by Penrose (1995) and the evolutionary economics school represented by Nelson and Winter (1982) tend to believe that the core advantage of an enterprise in the production process is not simply to avoid transaction costs, but the unique advantage of controlling a particular economic activity logically that quite different from the market behavior. Chen (2003) first proposed the concept of residual value of Cluster, but it refers to an individual enterprise within a cluster rather than the cluster as a whole, and the "residual value of Cluster" is defined as the extra benefit that an enterprise can achieve by joining a cluster than by not joining. Li and Yang (2005) pointed out that cluster residual value is the residual effect of an enterprise joining or not joining the cluster on the cluster itself. Chen and $\mathrm{Wu}(2005)$ argued that the benefits and costs of a cluster can be as tools for analyzing the cluster residual value. Although this finding is further analyzed the residual value of cluster itself than others, it is still based on whether an enterprise joins a cluster and how much the residual value of the cluster it can get if it does.

Based on the research gap, there are three problems could be summarized as below:

1) The analysis of producing cluster residual effect is insufficient.

2) The allocation mechanism of cluster residual value is lack of researches.

3) The influence of cluster internal network on cluster residual value is lack of researches.

\subsection{Research Objective}

In order to clearly understand the relationship between MSMEs and MSMEs' cluster and the allocation mechanism of cluster residual value, the research objectives of this research are set as below:

1) To explicate the influence mechanism between MSMEs and MSMEs' cluster.

2) To explicate the generation mechanism of cluster residual value.

3) To determine whether there is a significant effect of structural hole on allocating the residual value of MSMEs' cluster.

\subsection{Conceptual Framework}

The conceptual framework was developed from the literature review on structural hole, enterprise cluster, and cluster residual value. According to Foghani, Batiah, and Rosmini (2017) and many other scholars' studies, the clustering of MSMEs has been an inevitable trend of economic development in different economic areas. MSMEs clustering can not only distribute more benefits to MSMEs than they are individually in the market, but also can more in line with the higher efficient division of labor pattern required by global economic integration.

Although the cluster provides more value to its MSMEs, still many MSMEs are difficult to survey and grow in the fierce market competition. Therefore, this paper introduced the theory of structural hole to discuss the influence of residual value of MSMEs' cluster on the relationship between the growth of MSMEs and the cluster 


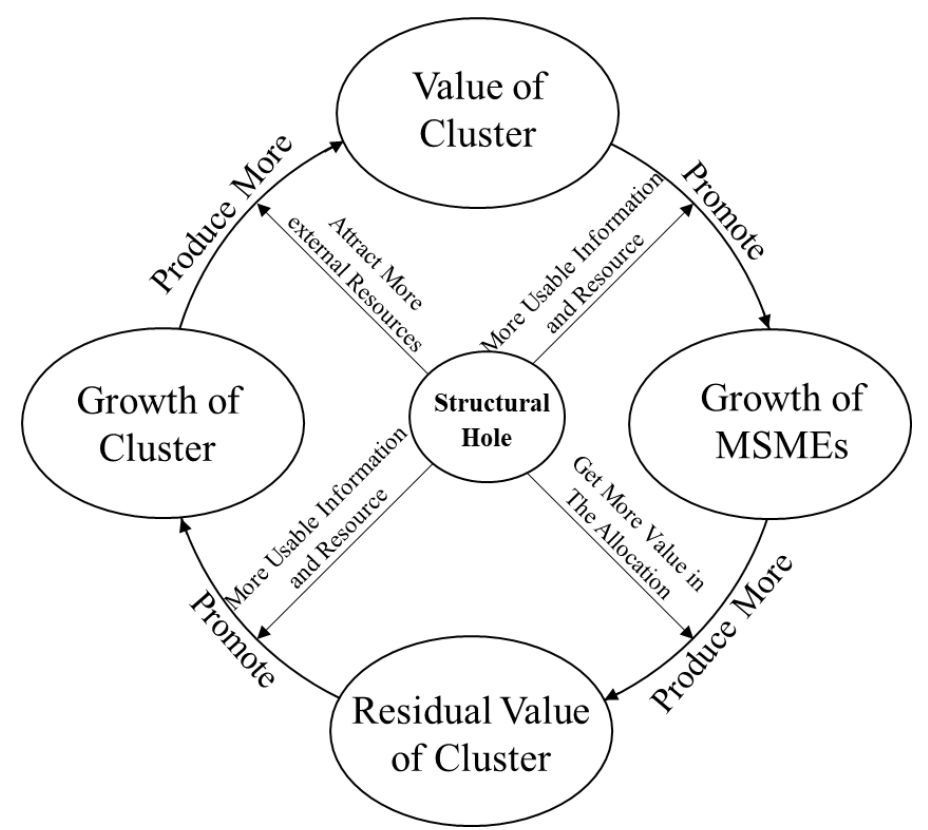

Figure 1. The Influence of Structural Hole on The Relationship Between MSMEs and MSMEs' Cluster. (developed by the author)

Enterprise is not an isolated unit it cannot exist without connection with other organizations or individuals. Therefore, an enterprise in a cluster is in two different networks at least. One is the tight internal network of the cluster, and the other one is the loose external social network relatively. Coleman (1990) noted that the tight network can generate cohesion, which can relatively make the high trust among network members, and the network emphasizes the common behavioral norms and concerted actions, which are conducive to the information sharing of network members and the common defense of risks.

However, Burt (1992) argued that the tight network could make its members all have the same or similar information, which causes a lack of information diversity and redundancy information among the members. As a result, the value of this network is reduced. The information that exists in the structure hole is likely to heterogeneous and non-redundant, which ultimately can bring the structure hole owner to have more value than other members in this network. Moreover, Burt (2004) argues that "opinion and behavior are more homogenous within than between groups, so people connected across groups are more familiar with alternative ways of thinking and behaving..." (pp. 349-350). In tightly networked clusters of people, discussion of redundant information is likely to be prevalent, therefore, the holes around and between these tight networks where an individual is also likely to find the greatest benefit in bridging the structural hole to another individual and/or network to access non-redundant information and/or resources. (Cowan \& Jonard, 2007). Therefore, Figure 1 illuminated that the value of cluster can help its member enterprises to develop, but only those who occupied structural holes in networks can obtain more resources and more competitiveness, so as to achieve faster growth. (Suo, 2019).

Enterprises exist in an uncertain cluster network and the agreements in the cluster network are unperfect, thus the profits of enterprises from the cluster network are not a constant, which is affected by the uncertain factors of enterprises themselves. The behaviors of organizations in the cluster, including enterprises, governments, intermediaries, and other investors, could have external influences inevitably. Therefore, they can't get a predictable fixed reward for their investment. As a result, as the figure 1 showed, a mature enterprise that occupied structural holes will tend to attract more enterprises to enter the cluster, and guide other enterprises in the cluster to invest in the cluster network and creating more residual value of the cluster because they can obtain more benefits in the process of allocating cluster's residual value by the structural holes they occupied.

Since the residual value of the cluster increases, it not only that the member enterprises can get more benefits, but also attract more enterprises to join the cluster, thus it promotes the growth of the cluster, as figure 1 demonstrated. In this process, the cluster can be considered as a node in a social network. The structural holes that the cluster occupied are the cluster's member enterprises occupied in the social network. Therefore, the cluster can get more usable information and resources to support its growth from these structural holes. 
During the cluster is growing up, the structural holes that it occupied in the social network can provide more resources to help to produce more value for its member enterprises. Finally, as figure 1 illustrated that the structural hole can help the cycle running positively.

Based on the above analysis, this paper proposes the following 4 hypotheses.

$\mathrm{H}_{1}$ : There is a significant relationship between cluster's value and growth of MSMEs.

$\mathrm{H}_{2}$ : There is a significant moderating effect of structural hole on the relationship between cluster's value and growth of MSMEs.

$\mathrm{H}_{3}$ : There is a significant relationship between structural hole and allocation of cluster's residual value.

$\mathrm{H}_{4}$ : There is a significant relationship between structural hole and growth of cluster.

\section{Literature Review}

\subsection{Enterprises' Cluster and Cluster's Value}

Scholars have different views on enterprise clusters from different perspectives: Becattini (2002) believes that enterprise clusters are the geographical gathering of companies and institutions that form interconnections in a particular industry. Humphrey and Schmitz (2002) believe that the enterprise cluster is the geographical concentration of enterprises' departments. A cluster can make an enterprise gain benefits that a single enterprise cannot get, which comes from collective efficiency. Van Dijk and Rabellotti (1997) consider enterprise clusters to be a group of enterprises that are close in spatial location and specialize in producing the same or similar products. Porter (1998) defines the concept of enterprise cluster as: "A geographical cluster of interconnected enterprises and institutions in a particular field. Clusters include a range of related industries and other competition-related entities, such as suppliers of components, machinery, equipment and services, suppliers of specialized infrastructure, etc. Clusters also tend to extend downstream to sales channels and customers, and horizontally to manufacturers of complementary products that are technically or technically related or have a common investment Products. In addition, many clusters also include government and other institutions for training, education, information, research, and technology." Uzor (2004) believes that enterprise clusters are small enterprises that provide similar products or services and operate in the same place. They cooperate and compete with each other, learn from each other, formulate common strategies to meet external challenges, and expand the market through the development networks.

According to the conceptions of value chains and value networks, enterprise clusters' value chain can be expressed as a special weighted flowing chain composed of nodes and arcs. (Zhao, 2008) Nodes refer to various actors, including enterprises, governments, universities, scientific research institutions, intermediary organizations, etc. Arc refers to the link connecting exchanges, transactions, or cooperation among nodes, including various input-output links, service links, sales links, joint research links, cooperative development links, etc. weighted flowing chain refers to the material, knowledge, information, etc. flowing on the arc. The value network of enterprise cluster refers to the intricate network system formed by the vertical and horizontal connection between different value chains in the cluster. The enterprise cluster value network inherits the element structural characteristics of the enterprise cluster value chain and forms a new kind of directed graph with special empowerment. The enterprise cluster value network is similar to the general network in that it is also composed of nodes and arcs, with inflows and outflows (or input and output) at the nodes, and flow at the arcs. The difference is that the nodes in the value network of enterprise clusters are not the same, they could be enterprises, intermediaries, research institutions, etc. The process of inflow and outflow at each node is the process of value creation or value appreciation at the node. The amount of inflow and outflow at each node is generally different. After passing a node, the material, knowledge, or information will change.

Therefore, based on the context of enterprises' cluster and cluster' value, enterprises as nodes embedded in the network of a cluster. The capital value of the cluster is the total amount of fluid substances on the arc, like information, knowledge, relationship benefits, etc. Enterprises or individuals in the cluster's network, they produce these fluid substances and also get benefit from it. As a result, the working efficiency of a cluster is dependent on the amount of fluid substances and the utilization ratio.

\subsection{Structural Hole}

Structural hole is a concept from social network research, originally developed by Ronald Stuart Burt. (Burt, 1992). Burt introduced this concept in an attempt to explain the origin of differences in social capital. Burt's theory suggests that individuals hold certain positional advantages or disadvantages from how they are embedded in neighborhoods or other social structures. A structural hole is understood as a gap between two 
individuals who have complementary sources to information. Structural holes exist in social networks when there is a lack of direct contact or tie between two or more entities (Burt, 1992). The theory of structural holes was developed to explain how to benefit from competition in social networks and their intersecting relationships (Burt, 1997). Burt took the perspective of how does a player gain competitive advantages in the competitive arena as the starting point of the research, and pointed out the social structure of the competition arena is the key factor to influence the rate of return on investment. However, homogeneous and reduplicative networks cannot lead to a social capital increase, therefore, the theory of structure hole was forming for explaining this question. Structural holes differ from "weak ties as bridges" as described by Granovetter (1973, p. 1065) in that it is not about the strength of the relationship between two entities but rather about the "chasm" or lack of a tie between entities (Burt, 1992).

The structure hole can be described as the phenomenon that one or some individuals in the social network have a direct connection, indirect connection, or disconnection with other individuals in the social network. As shown in figure 2.1, the network has four participants in A, B, C, D, of which there are no links among B, C, and D, but they are associated with A respectively. Thus, an occupied the central position in the network, the other three participants must pass A to connect each other, so A occupied three structural holes of $\mathrm{BC}, \mathrm{BD}$, and $\mathrm{CD}$, as a result, an obtain competitive advantages. Obviously, the structural hole is a chasm among the non-redundant contacts; it is a buffer, just like an insulator in a circuit.

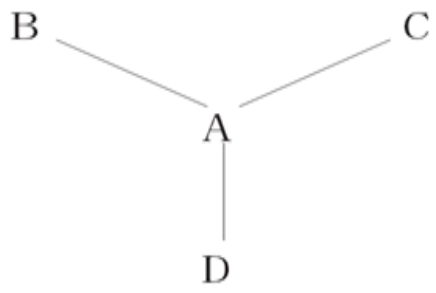

Figure 2.1. Structure Hole Illustrating (designed by author)

Because of structure holes, the contacts on both sides of the hole can bring cumulative rather than overlapping network benefits to the structure hole occupier. This includes information benefits and control benefits. The information benefit is realized mainly by access, timing, and referral. The controlling benefit refers to the fact that a third party can bridge between the two parties and decide which one should be taken into consideration first. Information is undoubtedly the essence of controlling benefits. This is similar to Simmel's idea of Tertius Gaudens. As Burt (2001) said, thoughts and behaviors of in-group members are more homogeneous than out-group members are, so people across groups are more familiar with alternative thoughts and behaviors to gain more choices and opportunities. The advantage of such thoughts and opportunities is the mechanism of brokerage becoming to social capital.

At present, when scholars in different fields use social network analysis to study network structure, the measurement of network structure is mainly divided into three levels, which are single enterprise, enterprise cluster, and the complete social network. (Suo, 2019). In the first level, scholars study a single enterprise in the form of network nodes. The centrality of network location is often selected as the measurement index of the competitiveness and the growth of an enterprise. In the second level, the link relations among industries or organizations within one industry are the core research content. Scholars study the positions of enterprise clusters or industries in the network and take network span, structure hole, and network heterogeneity as the test indexes for enterprises to acquire knowledge resources and improve their competitiveness. The third level research extends further to the overall social network. This is a more macroscopic perspective, which usually takes network density and network scale as the measurement indexes of the social network structure and the development of a society.

Due to this research focuses on the influence of structural hole on the growth of enterprises, allocation of residual value, and the cluster's development, the analysis is at the first and second levels. If an enterprise or a cluster in the position of a network bridge, it can not only have access to more information resources, which are not easily accessible by other network members, but also can obtain more heterogeneous resources. At the same time, it can control the flow direction of the network benefits. 


\subsection{Residual Value of Cluster}

In a cluster network, an individual or an organization can't obtain a predictable fixed reward for its investment behavior, and this investment behavior itself will have an external impact. (Hu, Wang, \& Wang, 2010) Hence, from the perspective of network relationship, the cluster's residual value reflects the total value of individual or organizational behavior, which is the sum of the value newly created by the members in the cluster. It is the value-added gained by individuals or organizations themselves and the value-added brought to other individuals or organizations. This view of residual value reflects the nature of cooperation and complementarity among individuals or organizations in the cluster network, sharing the risks and the benefits of the cluster development.

According to (Hu, etc., 2010), assumptions in the cluster network, the benefit of investment of an enterprise (i) in the cluster network to the enterprise itself is $\mathrm{R}_{\mathrm{i}}$; The benefit brought to individuals (including free workers) in the cluster network is $\mathrm{w}$ The revenue to the local government is $\mathrm{R}$; The income brought to other enterprises, such as enterprise $(j)$, is $R_{j}$, the total amount of residual value $(R)$ of the whole cluster is:

$$
R=R i+w+r+R j .
$$

(Formula 1)

In formula 1 , the amount of residual value $\mathrm{R}$ could be negative, it means someone in the cluster or the whole cluster has to take this deficit. It is assumed that the behavior of the enterprise's investment in the network has no external impact on the cluster, that is:

$$
\mathrm{R}=\mathrm{R}_{\mathrm{i}} \text {. }
$$

The enterprise's investment behavior only generates the residual value for the enterprise, that is, the cluster residual value is the enterprise's residual value. If the behavior of enterprise (i) investing in the network does not bring any impact to the government and individual operators, namely the external economy only affects the enterprise, such as technology shift between enterprises, the formula 1 is:

$$
\mathrm{R}=\mathrm{R}_{\mathrm{i}}+\mathrm{R}_{\mathrm{j}}
$$

(Formula 3)

In the cluster network, the investment behavior of enterprise $\mathrm{i}$ will inevitably bring uncertain influence to other enterprises in the network, it means there is a positive or negative impact on other enterprises in the cluster, then the formula can be expressed as:

$$
\mathrm{R}=\mathrm{R}_{\mathrm{i}}+\mathrm{R}_{\mathrm{j} 1}+\ldots+\mathrm{R}_{\mathrm{j}(\mathrm{n}-1)} 1<\mathrm{j} \leq \mathrm{n}
$$

In the cluster, if the regional market of the cluster is fixed, assuming that the future demand $\mathrm{Q}$ of the whole cluster is constant and the price $\mathrm{P}$ is fixed, then the revenue $\mathrm{R}$ of the whole cluster is fixed. Any enterprise can only share a variable reward if it cannot get a fixed reward for its input. Suppose the share ratio of enterprise (1), enterprise (2), .., enterprise (n) are $\beta_{1}, \beta_{2}, \ldots, \beta_{n}$, and $\beta_{1}+\beta_{2} \ldots+\beta_{n}=1$, in which the residual value that the enterprise (i) can get from the cluster is:

$$
\mathrm{R}_{\mathrm{i}}=\beta_{\mathrm{i}} \mathrm{R} \text {. }
$$

(Formula 4)

When enterprises are located in different positions in the cluster network, their $\beta$ values are different, so the residual value of the clusters allocated is also different. Generally, enterprises with a better location of structural holes can allocate more surplus value.

\section{Research Methodology}

The research design for this research was designed as quantitative research adopting survey questionnaires to collect the data from respondents who are entrepreneurs or high-level managers of MSMEs in China. The methodology of this research was designed on the basis of the available literature and practices of scholars, which is very important for appropriate research methods to obtain effective and reliable findings. It aims at reaching the objectives of this study.

In order to test the research hypothesis, a quantitative research design was employed to collect the primary data with questionnaire survey from 486 entrepreneurs or high-level managers of MSMEs. In this paper, KMO and Cronbach's Alpha was employed for testing the validity and reliability of all scale factors and variables. Pearson Correlation was adopted for testing the correlations between variables, and employing One-Way ANOVA to further specifies the direction of correlation and studies whether there is a causal relationship between variables.

\subsection{Population and Sample Size}

There are 23,280,312 MSMEs have registered on State Administration for Market Regulation. (SME Finance, 2019). Therefore, the sample size for the study is 400 derived from Taro Yamane formula (1967), but for more convenience and to protect from the incomplete questionnaire, the researcher used 500 samples in this research for easy computation. 


\subsection{Data Collection}

In order to avoid possible misunderstandings caused by different personal cognition about our designed questions, we distributed questionnaires in designated groups which formed by entrepreneurs or high-level managers of MSMEs, such as SME co-operation working places, entrepreneur social communities, activities, and meetings of "start-ups and innovation", and part-time MBA programs in universities.

\subsection{Reliability and Validity Tests of Questionnaire Design}

The questionnaire was tested with 30 people who are entrepreneurs or high-level managers of MSMEs, and they were not in the sample group. The reliability value was calculated by using Cronbach's alpha to ensure whether there was internal consistency within the items. The Alpha value of this pilot test was 0.918 , it indicated that the questionnaire of this research was highly reliable.

The index of item-objective congruence (IOC) is adopted in this study to test development for evaluating content validity at the item development stage (Rovinelli, \& Hambleton, 1977). The result of the IOC test was 0.745 , the designed questions of this research are acceptable.

\section{Research Findings and Analysis}

\subsection{Descriptive Analysis Results}

This research focuses on MSMEs in China, all data from 25 large size enterprises of 500 samples had been deleted, as a result, the total valuable sample number is 475 . The findings from this research illustrated that the majority of the respondents were male 54.8 percentage and female 45.2 percentage. The rate of female and male are not nearly balanced that could be caused by the survey target population is entrepreneurs or high-level of MSMEs. In addition, there are around 95.2 percent of respondents are entrepreneurs or high-level managers and having more than three-year working experience in their enterprise.

\subsection{Validity and Reliability Tests}

The validity test of this study is based on factor analysis. KMO is adopted to examine whether the collected data of this research are suitable for factor analysis, and to estimate whether the different measurement items under the same variable can reflect the characteristics of the measured variable more accurately.

The reliability test is a measurement method used to check whether the data from the questionnaire survey has consistency or not, which usually employ Cronbach's Alpha to test the consistency coefficient.

Table 1. Validity and Reliability Test Result

\begin{tabular}{lccc}
\hline Factors & Alpha & KMO & Sig \\
\hline Cluster's Value & .951 & .915 & $.000^{* * *}$ \\
Structural Hole & .983 & .985 & $.000^{* * *}$ \\
Growth of MSMEs & .956 & .918 & $.000^{* * *}$ \\
Allocation of Cluster's Residual Value & .962 & .917 & $.000^{* * *}$ \\
Growth of Cluster & .956 & .912 & $.000^{* * *}$ \\
All Scale Factor (25 Items) & .973 & .979 & $.000^{* * *}$ \\
\hline
\end{tabular}

Sig $<0.05$

The results of Table 1 revealed that all data of Independent Variables (Cluster's Value and Structural Hole,), Moderating Variable (Structural Hole), and Dependent Variables (Growth of MSMEs, Allocation of Cluster's Residual Value, and Growth of Cluster) have high validity and reliability. Thus, correlation and regression analyses can proceed.

\subsection{Correlation and Regression Analysis}

As the premise of regression analysis, Pearson Correlation is used to preliminarily judge whether the interaction and influence between variables exist and whether the hypothesis is valid in this research.

One-Way ANOVA as regression analysis in this paper is to further specify the direction of correlation and studies on whether there is a causal relationship between independent and dependent variables. 
Table 2. Coefficients of Correlation and Regression between Variables

\begin{tabular}{cccccccc}
\hline IV & DV & $\begin{array}{c}\text { Person Correlation } \\
\text { Coefficient }\end{array}$ & Adjusted $\mathrm{R}^{2}$ & $\mathrm{~F}$ & $\mathrm{t}$ & $\begin{array}{c}\text { Standardized } \\
\text { Coefficients Beta }\end{array}$ & Sig. \\
\hline CV & GM & .852 & .725 & 112.036 & 32.816 & .852 & .000 \\
SH & ACRV & .901 & .812 & 108.642 & 41.980 & .901 & .000 \\
SH & GC & .918 & .842 & 126.991 & 46.713 & .918 & .000 \\
\hline
\end{tabular}

Sig $<0.05$

Annotation: IV is Independent Variable; DV is Dependent Variable; CV is Cluster's Value; SH is Structural Hole; GM is Growth of MSMEs; ACRV is Allocation of Cluster's Residual Value; GC is Growth of Cluster.

Table 2 shows that the two-tailed test the correlations between independent and dependent variables are all at significant level (error-tolerant < 0.01). Values of Pearson Correlation are greater than 0.8, which indicates that there are high degrees of correlations between independent variables and dependent variables. As a result, hypotheses $\mathrm{H}_{1}, \mathrm{H}_{3}$, and $\mathrm{H}_{4}$ were accepted. Moreover, according to the coefficients of Adj. $\mathrm{R}^{2}$, there are high explanations percentage from cluster's value to the growth of MSMEs; from structural hole to allocation of cluster's residual value; from structural hole to growth of cluster. The standardized coefficients Beta here indicate that $\mathrm{H}_{1}, \mathrm{H}_{3}$, and $\mathrm{H}_{4}$ were accepted with positive relationships.

\subsection{Effective Test of Moderating Variable}

The effectiveness of the moderating variable can be analyzed by the method of variable standardization that is introduced in Aiken and West (1991). The variable standardization is a technique of mean centering, the purpose of it is to avoid the collinearity. After the regression analysis between the independent variables and the dependent variables under conditions of high and low score groups of the moderating variable, the influence result of the moderating variable on the relationship between the independent variables and the dependent variables can be presented. (Kraemer \& Blasey 2004). Table 3 shows the results of regressions on dependent variable (growth of MSMEs) into z score of independent variable (cluster's value); into z score of moderating variable (Structural hole); into the multiplication of $\mathrm{z}$ score of cluster's value and $\mathrm{z}$ score of structural hole.

Table 3. Regressions between Z Score of Variables

\begin{tabular}{|c|c|c|c|c|c|c|c|c|}
\hline & \multirow[b]{2}{*}{ Model } & \multicolumn{2}{|c|}{$\begin{array}{l}\text { Unstandardized } \\
\text { Coefficients }\end{array}$} & \multirow{2}{*}{$\begin{array}{c}\text { Standardized } \\
\text { Coefficients } \\
\text { Beta }\end{array}$} & \multirow[b]{2}{*}{$\mathrm{t}$} & \multirow[b]{2}{*}{ Sig. } & \multicolumn{2}{|c|}{ Collinearity Statistic } \\
\hline & & B & Std. Error & & & & Tolerance & VIF \\
\hline \multirow{3}{*}{1} & (Constant) & 3.470 & .031 & & 112.542 & .000 & & \\
\hline & Zscore $(\mathrm{CV})$ & 1.013 & .031 & .852 & 32.816 & .000 & 1.000 & 1.000 \\
\hline & (Constant) & 3.470 & .025 & & 141.137 & .000 & & \\
\hline \multirow[t]{3}{*}{2} & Zscore $(\mathrm{CV})$ & .483 & .042 & .406 & 11.386 & .000 & .336 & 2.973 \\
\hline & Zscore $(\mathrm{SH})$ & .650 & .042 & .547 & 15.319 & .000 & .336 & 2.973 \\
\hline & (Constant) & 3.508 & .035 & & 100.219 & .000 & & \\
\hline \multirow{3}{*}{3} & Zscore $(\mathrm{CV})$ & .463 & .044 & .390 & 10.444 & .000 & .307 & 3.260 \\
\hline & Zscore $(\mathrm{SH})$ & .638 & .043 & .536 & 14.782 & .000 & .324 & 3.084 \\
\hline & $\mathrm{CVxSH}$ & .246 & .031 & .405 & 1.509 & .000 & .593 & 1.686 \\
\hline
\end{tabular}

All z scores of variables are at significant level $(<0.05)$, which means the effect of moderating variable (Structural Hole) was significant. Therefore, hypothesis 2 was accepted. The following figure 4 shows the comparison of the regression effect of the high-value and low-value grouping of the moderating variable on the relationship between independent and dependent variables. 


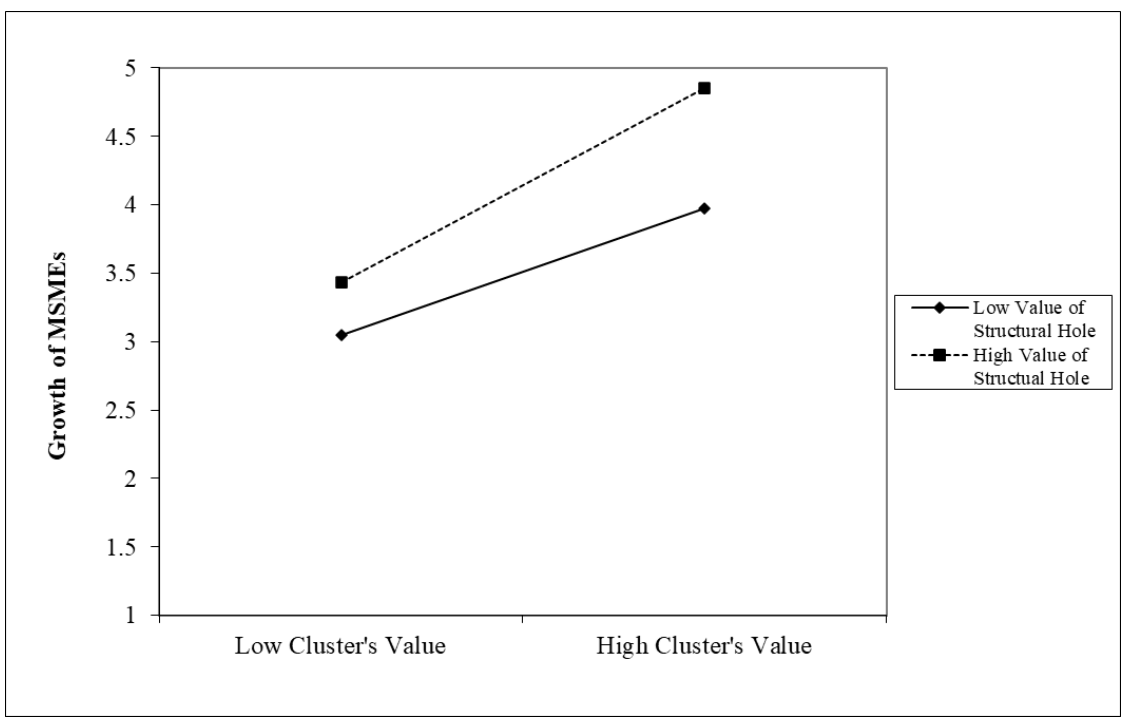

Figure 4. Comparison of regression effects of different groupings of moderating variable

The figure 4 displayed that the slopes of two lines are different obviously, which means the effects of Structural Hole as moderating variable on the correlation between the independent variable (Cluster's Value) and dependent variable (Growth of MSMEs) are different with high and low value groups of cluster's value. It demonstrated the effect of the moderating variable is significant, and the $\mathrm{H}_{2}$ was accepted.

To sum up, after reliability, validity, correlation, and regression analyses, a conclusion can be drawn that hypothesis 1,3 , and 4 were accepted with positive correlations. In addition, by the $\mathrm{z}$ score variables regression analysis, hypothesis 2 was accepted.

\section{Research Contribution}

\subsection{Contribution to Knowledge}

This research discussed the relationship among cluster's value, growth of MSMEs, allocation of residual value, and growth of cluster, it revealed an intrinsic value of cluster's network, providing a new perspective of innovation for future research. According to the findings of this research, it expounded that the differences of cluster's residual value allocation could be influenced by the structural hole significantly, and because of it, the structural hole also has moderating effect on the relationship between cluster's value and growth of MSMEs. On the premise that there are positive correlations between structural hole and enterprise growth, and between enterprise growth and cluster growth, this paper verifies that there is also a positive correlation between structural hole and cluster growth, and provides a valuable theoretical perspective on the development of MSMEs and MSMEs' cluster. Moreover, by the analysis of the cluster's residual value allocation, this study also provides a theoretical reference for the Cluster's MSMEs in how to occupy and use structural hole to gain more residual value of the cluster. To compare with previous studies, this research presents a new perspective on the development of cluster based on its internal network, which could help MSMEs to utilize of the residual value of the cluster maximumly, so as to obtain the growth of MSMEs and the cluster.

\subsection{Contribution to Practice}

In daily social communicational activities, enterprises should focus on expanding the scope of their social circle and network, and devote the time and the energy to establishing connections with individuals and social groups at different levels and fields, such as governments, media, and financial institutions. MSMEs should constantly look for structural holes in their networks, dig unique resources, and enrich new knowledge by participating in market and non-market activities. Entrepreneurs should also focus on selecting the "primary contacts" who are most important to the relationships and use them to gain access to a wider range of internal and external network of the cluster. This approach could improve the efficiency of relationship connection and maintain a balance among connections, which could be differentiation, decentralization, and clusterization. Moreover, in order to identify the relationship that can really bring innovation opportunities, MSMEs should consciously sort out connections with different levels in the relationship network at ordinary times. If the structural hole is in an active position, the enterprise can use the structure hole to seize the usable and valuable resources, otherwise, if it is in a passive position, such as the number of structural holes is not enough or very lack, the enterprise also should actively "create" opportunities for enriching its value. 
Furthermore, the dynamic change is accompanied by growth, management, thus, optimization and reconstruction of the structure hole are important for MSMEs in relationship management. MSMEs could have to reach the different needs for its survival and development by the dynamic adjustment of structural holes with different characteristic forms, diversity, and heterogeneity. On the one hand, MSMEs need to get rid of their own existence of various business difficulties, must proceed from the individual entrepreneur and employees, who should have a good performance on innovation, marketing strategy, satisfying customer needs, winning in the competitive market, and obtaining sustainable profits. On the other hand, when the enterprise is faced with the current situation of limited knowledge and marketing practice, and the effect of weakening from competitors, it should flexibly use the occupied structural hole in the cluster's network to explore better and richer benefits of the cluster. In the process of construction and maintenance of individual relationship networks, MSMEs need to pay more attention to searching structure holes in the network of different groups.

Clusters should also initiatively enhance the connections strength, the relevance, and the heterogeneity of the related networks, and enable differentiated networks' members to communicate and interact each other more closely and smoothly, which could help the cluster to reinforce the advantages of obtaining and controlling usable sources, so as to improve the level of innovation. At the same time, MSMEs should pay attention to prevent the disadvantageous situation that informal organizations and closed networks could disintegrate their structure hole. There are many ways, such as optimizing, eliminating, and reconstructing their redundancy relationship, which the enterprise could adopt to expand the diversity of its relationship network, explore and identify new potential opportunities, coordinate internal and external related parties, and rebuild the trust and the reputation in the market. Thus, the positive circulation as figure 1 described can run efficiently.

\subsection{Limitation and Future Research}

This study mainly focusses on the research about the influence of allocating the residual value of MSMEs' cluster on the growth of MSMEs and the cluster, however, with the development of the social economy and globalization, more and more enterprises clusters participate in the division of labor in the industrial chain, hence, more new functions of clusters are formed in the market nowadays, which could be studied in the future research. Moreover, the functions of cluster also could be deeper analyzed from the perspective of closed and opened internal network. In addition, allocation of residual value of cluster is touched upon in this study, which could be also deeper analyzed from the angle of creation and transfer in the future study. Although it is nearly impossible for one enterprise to occupy all structure holes in a network, the structure holes can help MSMEs to acquire more usable and valuable resources. However, too many redundancy structure holes could cost too much energy and reducing the work efficiency of MSMEs for maintaining them. Thus, how to optimize the relationship network and structure holes' location could be studied in the future.

\section{References}

Aiken, L. S., \& West, S. G. (1991). Multiple regression: Testing and interpreting interactions. Sage Publications, Inc.

Becattini, G. (2002). Del distrito industrial marshalliano a la teoría del distrito industrial contemporánea. Una breve reconstrucción crítica. Investigaciones Regionales, 1, 9-32.

Burt, R. S. (1992). Structural Holes. Cambridge, MA: Harvard University Press.

Burt, R. S. (1997). The contingent value of social capital. Journal of Administrative Science Quarterly, 42(2), 339-365. https://doi.org/10.2307/2393923

Burt, R. S. (2001). Bandwidth and echo: trust, information, and gossip in social networks. In A. Casella \& J. E. Rauch (Eds.), Integrating the Study of Networks and Markets. New York: Russell Sage Foundation.

Burt, R. S. (2004). Structural Holes and Good Ideas. American journal of sociology, 11(2), 349-399. https://doi.org/10.1086/421787

Chen, L. H., \& Wu, T. Z. (2005). Enterprise clusters and cluster's residual value. Journal of Economist, 2, 178-179.

Chen, X. M. (2003). Theory and practice of SMEs' cluster. Beijing: Economics and Science press.

Chinabgao. (2018). Analysis of the number of small and medium-sized enterprises in China. Retrieved September 11, 2018, from the Chinabgao website http://www.chinabgao.com/k/qiye/28142.html

Coleman, J. (1990). Foundations of Social Theory. Cambridge, MA: The Belknap Press of Harvard University.

Cowan, R., \& Jonard, N. (2007). Structural holes, innovation and the distribution of ideas. Journal of Economic 
Interaction and Coordination, 2(2), 93-110. https://doi.org/10.1007/s11403-007-0024-0

Foghani, S., Mahadi, B., \& Omar, R. (2017). Promoting Clusters and Networks for Small and Medium Enterprises to Economic Development in the Globalization Era. SAGE Open. https://doi.org/10.1177/2158244017697152

Granovetter, M. (1973). The Strength of Weak Ties. American Journal of Sociology, 78(6), 1360-1380. https://doi.org/10.1086/225469

Hu, D. F., Wang, L. P., \& Wang, W. (2010). Cluster residual and the source of cluster effect, based on structural holes theory. Journal of East China Economic Management, 24(11), 70-73.

Humphrey, J., \& Schmitz, H. (2002). How Does Insertion in Global Value Chains Affect Upgrading in Industrial Cluster. Journal of Regional Studies, 36, 1017-1027. https://doi.org/10.1080/0034340022000022198

Kraemer, H. C., \& Blasey, C. M. (2004). Centring in regression analyses: A strategy to prevent errors in statistical inference. International Journal of Methods in Psychiatric Research, 13(3), 141-151. https://doi.org/10.1002/mpr.170

Li, N., \& Yang, H. Q. (2005). Cluster residual and internal coordination mechanism of enterprise cluster. Nanjing: Nankai management review press.

Nelson R. R., \& Winter, S. G. A. (1982). Evolutionary theory of economic change. Cambridge: Cambridge University Press.

Penrose, E. (1995). The theory of the growth of the firm. Oxford: Oxford University Press. https://doi.org/10.1093/0198289774.001.0001

Porter, M. E. (1998). Clusters and new economics of competition. Harvard Business Review, 11, 77-92.

Rovinelli, R. J., \& Hambeton, R. K. (1977). On the use of content specialists in the assessment of criterion-referenced test item validity. Dutch Journal of Educational Research, 2, 49-60.

SME Finance Forum. (2020). SME in China. Retrieved June 11, 2020, from SME Finance Forum website https://www.smefinanceforum.org/search/node/SMEs\%20china

Suo, L., \& Bunchapattanasaakda, C. (2019). Influence of entrepreneurial structural hole in the growth of SMEs in Yunnan, China. Asian Journal of Science and Technology, 10(10), 10332-10340.

Uzor, O. O. (2004). Small and Medium Scale Enterprises Cluster Development in South-Eastern Region of Nigeria. Berichte aus dem Weltwirtschaftlichen Colloquium der universitat Bremen, Nr. 86. Institut fur Weltwirtschaft und Internationals management. Universitat Bremen.

Van Dijk., \& Rabellotti. (1997). Clusters and networks as sources of cooperation and technology diffusion for small enterprises in developing countries. Frank Cass \& Co. Ltd.

World Bank. (2019). Small and Medium Enterprises (SMEs) Finance. Retrieved June 11, 2020. From the World Bank website https://www.worldbank.org/en/topic/smefinance

Zhao, H. (2008). Study on the formation and aggregation of the value network of enterprises cluster. Chongqing: College of Economics and Business Administration Chongqing university press.

\section{Copyrights}

Copyright for this article is retained by the author(s), with first publication rights granted to the journal.

This is an open-access article distributed under the terms and conditions of the Creative Commons Attribution license (http://creativecommons.org/licenses/by/4.0/). 